Y.-H. Chu, N.B. Suntzeff, J.E. Hesser, and D.A. Bohlender, eds.

\title{
Recent Star Formation History of the Magellanic Clouds
}

\author{
Eva K. Grebel \\ UCO/Lick Observatory, UCSC, Santa Cruz, CA 95064, USA \\ Wolfgang Brandner \\ JPL/IPAC, Mail Code 100-22, Pasadena, CA 91125, USA
}

\begin{abstract}
.
A new age calibration of Cepheids and supergiants is used to study the large-scale recent star formation history of the LMC and the SMC. We find evidence for migration of star formation along the LMC bar as well as for the existence of long-lived $(\approx 200 \mathrm{Myr})$ extended star-forming features.
\end{abstract}

\section{Introduction}

Due to their proximity the Magellanic Clouds are well suited to study largescale star formation processes based on resolved stellar populations. The ongoing CCD surveys such as the Magellanic Clouds Photometric Survey and the EROS, $\mathrm{MACHO}$, and OGLE microlensing projects are only partially available, yet the databases maintained at the Centre des Données astronomiques de Strasbourg (CDS) and at the Astrophysical Data Center (ADC, NASA) contain already a wealth of information useful for this kind of study.

We are using data on Cepheids (Artiukhina et al. 1997) and non-variable supergiants in both Clouds to trace their recent large-scale star formation history. We carried out a new period-age calibration based on Galactic and Magellanic Cepheids in clusters by fitting Geneva isochrones to the cluster main sequences. The $U B V$ photometry of the non-variable supergiants (Rousseau et al. 1978; Isserstedt 1979, 1982a; Feitzinger \& Isserstedt 1983; Schmidt-Kaler et al. 1998) was dereddened using Isserstedt's (1982b) intrinsic colors for Magellanic supergiants. The supergiants were age-calibrated through Geneva evolutionary tracks.

The Cepheid age calibration covers an age range of roughly 230 to $30 \mathrm{Myr}$, and the supergiants trace the past 30 to $2 \mathrm{Myr}$. $230 \mathrm{Myr}$ correspond to one rotation period of the LMC. We visualized the resulting star formation history through animations to study its spatial variations with time.

\section{Results for the LMC}

Our data show that star formation was active in the bar region at distinct regions at certain times, while other areas were inactive. Star formation along the bar progressed from the southeast to the northwest. 
Only two large activity regions appear to have formed within the past $30 \mathrm{Myr}$ : 30 Dor and the surrounding supershells LMC 3 (simultaneously) and LMC 2 (a little later), and LMC $4 \& 5$. LMC 4 is the only supershell with a radial stellar age gradient as expected from self-propagating star formation in a spherically expanding shell. Our data contradict the suggested formation of 30 Dor through shock-shock collisions of LMC 2 and LMC 3 (Chernin et al. 1995).

Many of the areas coincident with today's supergiant shells have been active throughout the past $200 \mathrm{Myr}$ forming long-lived chains of star formation. There is no clear evidence of progression of star formation along these chains, which are vaguely reminiscent of spiral arms. The large-scale age structure does not support the clock-wise age increase suggested by the bow-shock scenario of de Boer et al. (1998). The long-lived chains are best reproduced by models that include the effect of an off-centered bar on a rotating disk (Gardiner et al. 1998).

\section{Results for the SMC}

The SMC shows a much more complex picture since it is not seen nearly face-on like the LMC, is strongly disrupted, and has a large depth extent along the line of sight. Our data show that the most recent star formation occurred mostly along the bar of the SMC, while slightly older populations show a more widespread distribution. Recent star formation is also visible along the beginning of the bridge between the Magellanic Clouds.

For both Clouds ages and positions of young star clusters agree very well with the star formation history as outlined by Cepheids and other supergiants.

Acknowledgments. We wish to thank Jörg Isserstedt whose impressive movie of the star formation history of the LMC inspired the current study. Terry Figel's (UCSC Vizlab) help in transferring our movie to video tape is gratefully acknowledged. EKG thanks Dennis Zaritsky for support through NASA LTSA grant NAG-5-3501.

\section{References}

Artiukhina, N.M., Durlevich, O.V., Frolov, M.S., et al. 1995, General Catalogue of Variable Stars, 4th ed., vol.V, Kosmosinform, Moscow

Chernin, A.D., Efremov, Y.N., \& Voinovich, P.A. 1995, MNRAS, 275, 313

de Boer, K.S., Braun, J.M., Vallenari, A., \& Mebold, U. 1998, A\&A, 329, L49

Gardiner, L.T., Turfus, C., \& Putman, M.E. 1998, ApJ, 507, L35

Feitzinger, J.V., \& Isserstedt, J. 1983, A\&AS, 51, 505

Isserstedt, J. 1979, A\&AS, 38, 239

Isserstedt, J. 1982a, A\&AS, 50, 7

Isserstedt, J. 1982b, A\&A, 115, 97

Rousseau, J., Martin, N., Prévot, L., Rebeirot, E., Robin, A., \& Brunet, J.P. 1978, A\&AS, 31, 243

Schmidt-Kaler, Th., Gochermann, J., Östreicher, M.O., et al. 1999, MNRAS, 306,279 


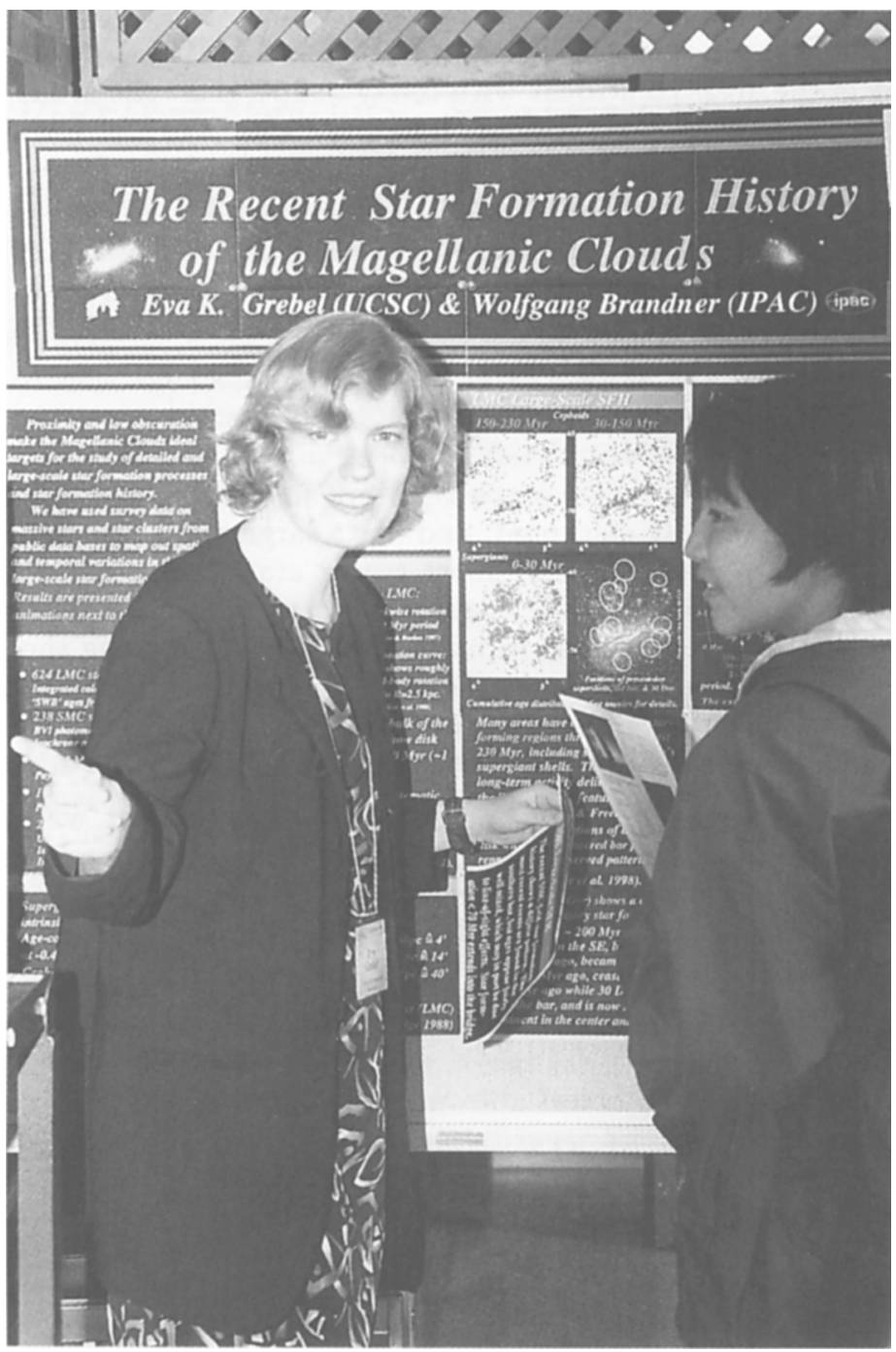

Eva Grebel would likely have won any award for most colourful poster! 